\title{
Novel Class of Probes for Multimodal Microscopy of Cells
}

Cesare De Pace ${ }^{1}$, Lorena Ruiz Perez ${ }^{1}$, Giovanni Bruno ${ }^{2}$, Xiaohe Tian $^{3}$ and Giuseppe Battaglia $^{4}$

${ }^{1}$ University College London (UCL), London, England, United Kingdom, ${ }^{2}$ Università di Bari, Bari, Puglia, Italy, ${ }^{3}$ Anhui University, Hefei, Anhui, China (People's Republic), ${ }^{4}$ University College of London (UCL); Institute for Bioengineering of Catalonia (IBEC); The Barcelona Institute for Science and Technology (BIST); Institució Catalana de Recerca i Estudis Avançats (ICREA), London, England, United Kingdom

Cellular imaging requires probes to increase the contrast of the cellular target under study due to the plethora of biomolecules within the cell. Generally, cellular probes provide contrast only for light or electron microscopy. Few probes bestow contrast for both confocal or electron microscopy, yet they may hinder cell functions due to their size, and/or not provide the expected resolution due to their distance from the target. Nanoscopic engineered organometallic probes meld tailored design and specificity for their target with compatibility for fluorescence and electron contrast. Hence, these probes enable correlative light and electron microscopy (CLEM) imaging $(1,2)$. The correlation between the two techniques is pivotal to compare the results from a novel technique such as liquid-phase electron microscopy (LPEM) of cells. Furthermore, organometallic probes offer the outstanding advantage of owning high electron density due to their metal-core, usually made by exogenous elements for the cells, such as iridium(1). Thus, this peculiarity may offer the possibility to analyse the elemental composition of the specimen with other complementary techniques. Energy-filtered transmission electron microscopy (EF-TEM) may further characterise the target of interest within the cell offering insight on the intercalation of the probe with its target. We used these compounds to analyse and image pivotal nuclear and cytosolic components of the cell aiming to reach an uncharted resolution within whole cells.

Organometallic probes have started to demonstrate their unique properties as contrast providing agents for microscopic techniques such as correlative light and electron microscopy (CLEM), energy-filtered transmission electron microscopy (EF-TEM), and liquid-phase electron microscopy (LPEM). Thus, compared to other labelling agents, the organometallic compounds may very well represent a novel class of microscopic probes, which can shed light to novel investigations on cellular mechanisms, and, ultimately, their associated diseases. 

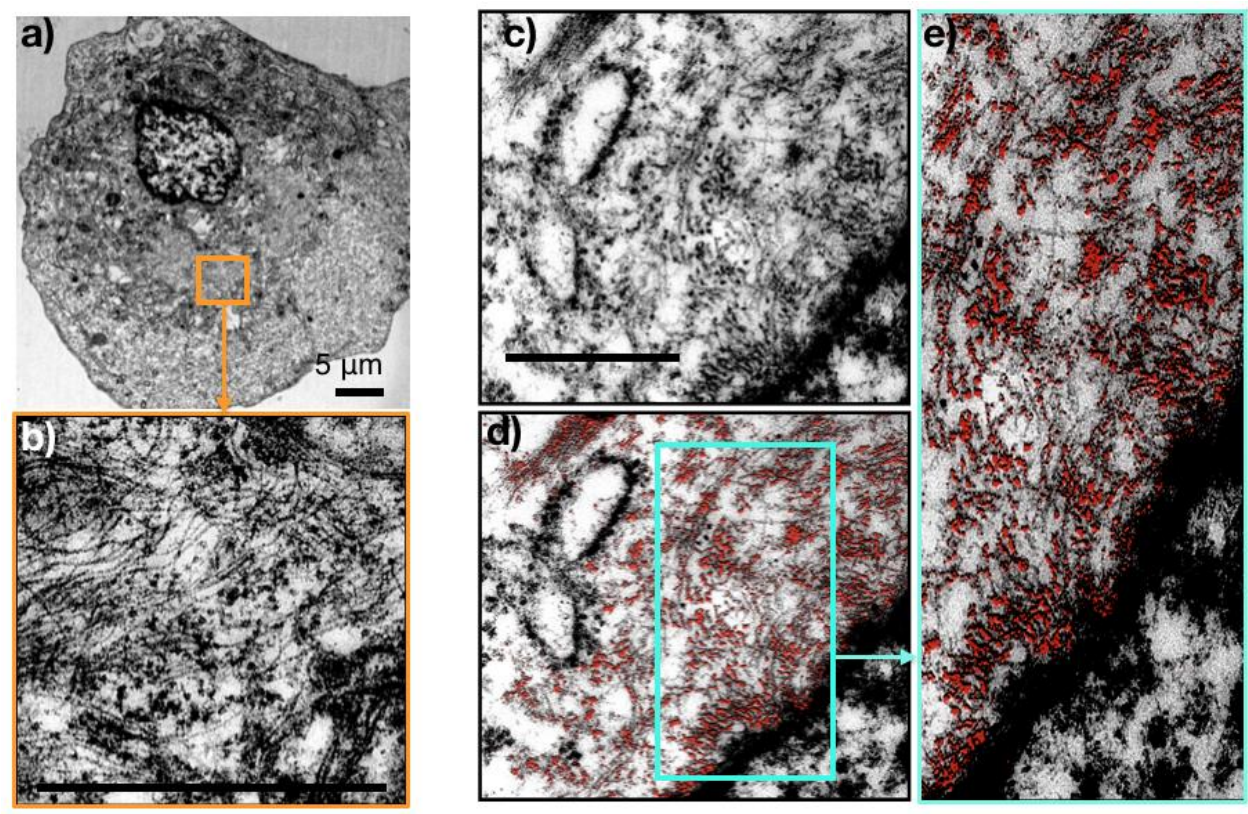

Figure 1. (a-e) EM micrographs of a cell stained by Ir-Tub and OsO4. d,e) CLEM micrographs displaying the confocal signal in red.

a) Gray scale

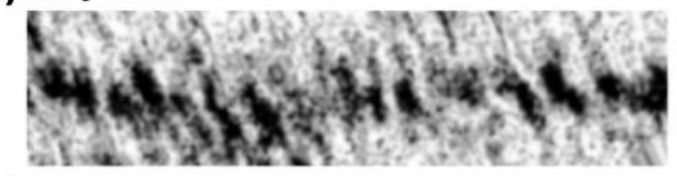

b) Iridium

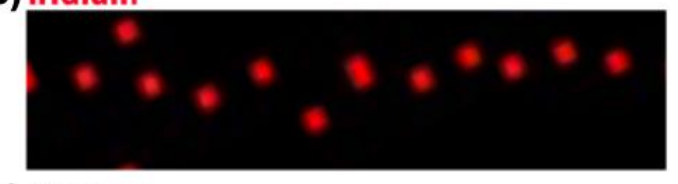

c) Merged

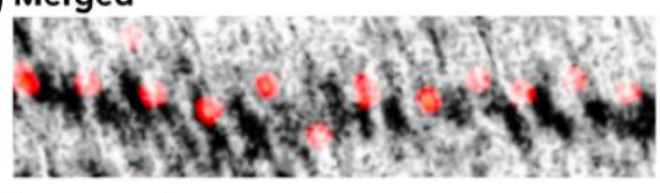

$10 \mathrm{~nm}$ d)

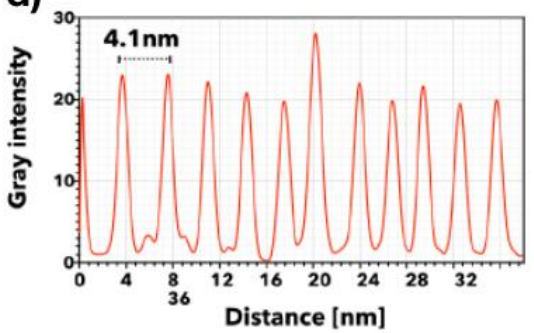

e)

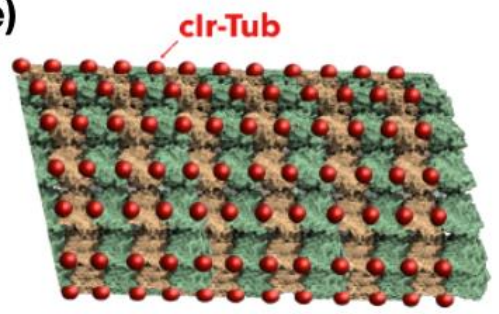

Figure 2. (a) EM micrograph displaying a cell stained by Ir-Tub, (b) its Ir elemental map and (c) their overlapping. The measurements of the Ir atoms distance (d) in the elemental mapping and (e) in silico model.

References

1. Tian, X. et al. An Organometallic Ir(III) Molecular Probe for Imaging Microtubules in Fluorescence and Electron Microscopy. bioRxiv (2018).

2. Tian, X., Hussain, S., De Pace, C., Ruiz-Pérez, L. \& Battaglia, G. ZnII Complexes for Bioimaging and Correlated Applications. Chem. - An Asian J. (2019). doi:10.1002/asia.201801437 